\title{
Accurate Localization in Combination with Planet Observation and Dead Reckoning for Lunar Rover
}

\author{
Yoji KURODA, Toshiharu KUROSAWA \\ Department of Mechanical Engineering, Meiji University \\ 1-1-1 Higashi-mita, Tama-ku, Kawasaki, Japan \\ \{ykuroda, ce22228\}@isc.meiji.ac.jp
}

\begin{abstract}
This paper proposes a localization method to estimate position and azimuth of a lunar rover. In the proposed method, the position is precisely estimated by integration of an absolute and a relative position. The absolute position is measured by observing the sun and the earth, and the relative position is determined by dead reckoning. Effectiveness is confirmed by the results of simulations. Moreover, differences between proposed method and Extended Kalman Filter are shown by results of long-distance simulation.
\end{abstract}

Keywords- Lunar Rover, Sensor Fusion, Dead Reckoning, Localization, EKF(Extended Kalman Filter)

\section{INTRODUCTION}

Recently, exploration missions on planetary or moon surface by using robotic rovers have attracted great deal of attention, because rovers could pick scientific information up directly from the surface [1]. Since we have to know where the information is collected, and to begin with, the rovers have to reach a destination, the rovers must know their position on the surface. Some methods to identify the position have been developed in the filed of mobile robots on the earth, e.g., GPS (Global Positioning System), map based localization, landmarks based localization, dead reckoning [5], etc. No methods are good enough for using on the moon or the other planets, because of the following reasons. GPS is not available because it needs at least four satellites in orbit. Detailed maps are not usually measured beforehand, therefore, map based localization cannot be used. In order to use landmarks based localization, we have to deploy plenty of landmarks on the surface of the moon or the other planets. Dead reckoning is not accurate enough when a robot moves for a long range, because of accumulating the integration errors [2-4].

This paper proposes a localization method to estimate the position and azimuth of a lunar rover. In the method, precise positioning information is obtained by a sensor fusion technique that is an integration of the absolute position and the relative position [5]. The proposed method is called "Localization in combination with Absolute and Relative positions (LAR)". The absolute position is calculated by observing the sun and the earth [6-8], and the relative position is measured by the dead reckoning. The effectiveness of LAR is confirmed by some numerical simulations.

\author{
Akiyoshi TSUCHIYA, Takashi KUBOTA \\ Institute of Space and Astronautical Science \\ Japan Aerospace Exploration Agency \\ 3-1-1 Yoshinodai, Sagamihara, Japan \\ kubota@nnl.isas.ac.jp
}

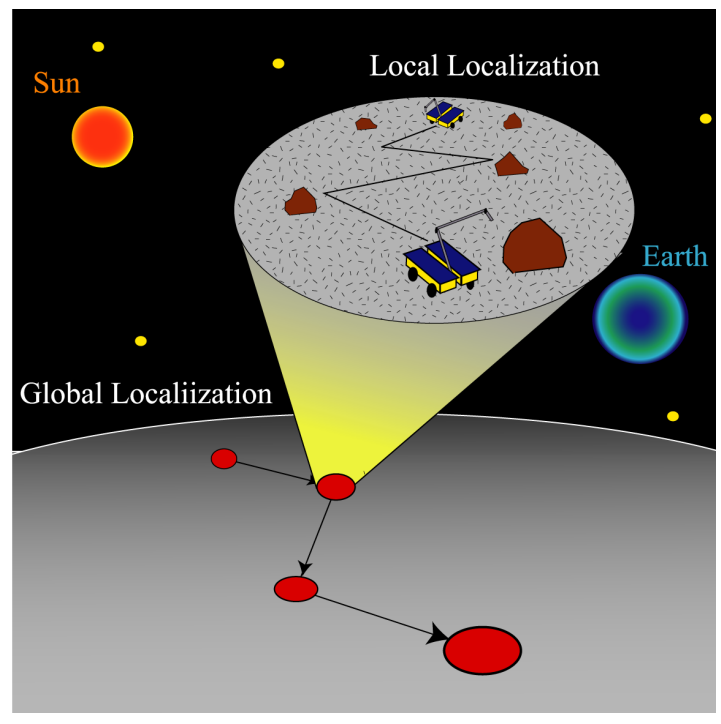

Fig. 1. Localization for lunar rover

\section{THE SUN AND EARTH OBSERVATION}

\section{A. Coordinates Systems}

In order to calculate the absolute position of the rover on the moon by observing the sun and the earth, the following four coordinates systems are used for.

CS1. Equatorial coordinates system at center of the earth

Z-axis: north celestial pole

$\mathrm{X}$-axis: vernal equinox direction

Y-axis: right-hand system of $\mathrm{x}-\mathrm{Z}$

Origin: the earth

The positions of the sun and the moon are defined by this coordinates system.

\section{CS2. Equatorial coordinates system at center of the moon \\ Z-axis: north celestial pole \\ $\mathrm{X}$-axis: vernal equinox direction \\ Y-axis: right-hand system of $\mathrm{x}-\mathrm{Z}$ \\ Origin: the moon}

We can calculate the position of the sun by transforming the coordinates system CS1 to CS2. 


\section{CS3. The moon fixed coordinates system}

Z-axis: north pole of moon

$X$-axis: meridian of the moon

Y-axis: right-hand system of $\mathrm{x}-\mathrm{Z}$

Origin: the moon

This coordinates system is changed into the coordinate which take the moon rotation into account from the inertia space fixed coordinates system CS2.

\section{CS4. Horizontal coordinates system of the moon}

\section{Z-axis: zenith}

$\mathrm{X}$-axis: south

Y-axis: east

Origin: center of the rover

This is the horizontal coordinates system which the rover uses on the moon, though the coordinates systems of CS1-CS3 are based on the celestial bodies.

The values directly observed by the sun and the earth sensors are described by direction cosine. But, CS4 is rectangular coordinate system. Thus, CS4 is redefined as the direction cosine indication. The altitudes of the sun and the earth are defined as $h_{s}$ and $h_{e}$, and their azimuths are represented by $A_{z s}$ and $A_{z e}$. Then, the positions of the sun and the earth are described as follows:

$$
\begin{gathered}
\left(\begin{array}{c}
\cos \left(h_{s}\right) \cos \left(2 \pi-A_{z s}\right) \\
\cos \left(h_{s}\right) \sin \left(2 \pi-A_{z s}\right) \\
\sin \left(h_{s}\right)
\end{array}\right)=\left(\begin{array}{l}
x_{s}(t) \\
y_{s}(t) \\
z_{s}(t)
\end{array}\right) \\
\left(\begin{array}{c}
\cos \left(h_{e}\right) \cos \left(2 \pi-A_{z e}\right) \\
\cos \left(h_{e}\right) \sin \left(2 \pi-A_{z e}\right) \\
\sin \left(h_{e}\right)
\end{array}\right)=\left(\begin{array}{l}
x_{e}(t) \\
y_{e}(t) \\
z_{e}(t)
\end{array}\right)
\end{gathered}
$$

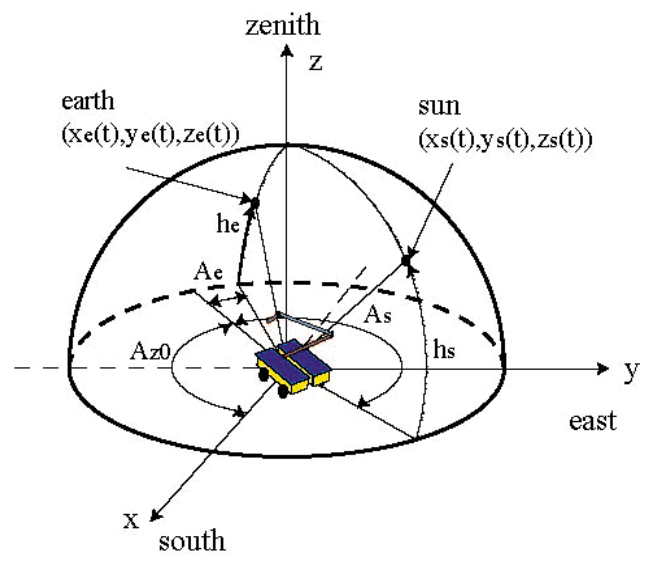

Fig. 2. Horizontal coordinates system

\section{B. Self-Position Estimation}

The rover finds its absolute position on the moon by observing the sun and the earth. Here the rover is supposed to have inclinometers and a precise time clock. The rover is also supposed to have moon orbital information.

Altitudes of the sun and the earth are obtained from a sun sensor and an earth sensor on the rover, respectively. Two of three attitude angles of the rover, roll and pitch, are obtained by inclinometers. On the other hand, the yaw angle of the rover cannot be directly measured because, a magnetic sensor is not available on the moon. Therefore, not only the position but also the yaw angle must be estimated by using the other angles, altitudes and azimuths of the sun and the earth.

Thus, unknown state is $x=\left(\lambda, \phi, A_{z 0}\right)$. Where $\lambda$ and $\phi$ represent longitude and latitude of the moon. $A_{z 0}$ represents the azimuth of the rover. By redefining $A_{z s}$ and $A_{z e}$ as $A_{z s}=A_{z 0}+A_{s}$ and $A_{z e}=A_{z 0}+A_{e}$ respectively, equation (3) and (4) can be rewritten as follows.

$$
\begin{aligned}
& \left(\begin{array}{c}
\cos \left(h_{s}\right) \cos \left(A_{z 0}+A_{s}\right) \\
-\cos \left(h_{s}\right) \sin \left(A_{z 0}+A_{s}\right) \\
\sin \left(h_{s}\right)
\end{array}\right)=\left(\begin{array}{l}
x_{s}(t) \\
y_{s}(t) \\
z_{s}(t)
\end{array}\right) \\
& \left(\begin{array}{c}
\cos \left(h_{e}\right) \cos \left(A_{z 0}+A_{e}\right) \\
-\cos \left(h_{e}\right) \sin \left(A_{z 0}+A_{e}\right) \\
\sin \left(h_{s}\right)
\end{array}\right)=\left(\begin{array}{l}
x_{e}(t) \\
y_{e}(t) \\
z_{e}(t)
\end{array}\right)
\end{aligned}
$$

When the observation by each sensor is performed $N$ times in $t=t_{0}, t_{1}, \ldots, t_{N-1}$, observed values are:

$$
\left(\begin{array}{l}
h_{s 0} \\
A_{s 0} \\
h_{e 0} \\
A_{e 0}
\end{array}\right)\left(\begin{array}{l}
h_{s 1} \\
A_{s 1} \\
h_{e 1} \\
A_{e 1}
\end{array}\right) \cdots\left(\begin{array}{l}
h_{s N-1} \\
A_{s N-1} \\
h_{e N-1} \\
A_{e N-1}
\end{array}\right)=y_{0}, y_{1}, \cdots y_{N-1}
$$

And, a state that should be estimated is:

$$
\hat{x}=\left(\hat{\lambda}, \hat{\phi}, \hat{A}_{z 0}\right)
$$

$\hat{x}$ is estimated from the observed values $\left\{y_{0}, y_{1}, \cdots, y_{N-1}\right\}$ by the least square method. If the true values of $y$ were defined as:

$$
\bar{y}=\left(\bar{h}_{s}, \bar{A}_{s}, \bar{h}_{e}, \bar{A}_{e}\right)
$$

evaluation function $J$ described by the following least square equation is introduced to find an optimal $y$. 


$$
\begin{aligned}
& J=\sum_{i=0}^{N-1}\left\{h_{s i}-\bar{h}_{s i}\left(\lambda, \phi, A_{z 0}, t_{i}\right)\right\}^{2} \\
& +B_{1} \sum_{i=0}^{N-1}\left\{A_{s i}-\bar{A}_{s i}\left(\lambda, \phi, A_{z 0}, t_{i}\right)\right\}^{2} \\
& +B_{2} \sum_{i=0}^{N-1}\left\{h_{e i}-\bar{h}_{e i}\left(\lambda, \phi, A_{z 0}, t_{i}\right)\right\}^{2} \\
& +B_{3} \sum_{i=0}^{N-1}\left\{A_{e i}-\bar{A}_{e i}\left(\lambda, \phi, A_{z 0}, t_{i}\right)\right\}^{2} \\
& \frac{\partial J}{\partial x}=0
\end{aligned}
$$

In order to solve the equation, we use the Newton -Raphson method.

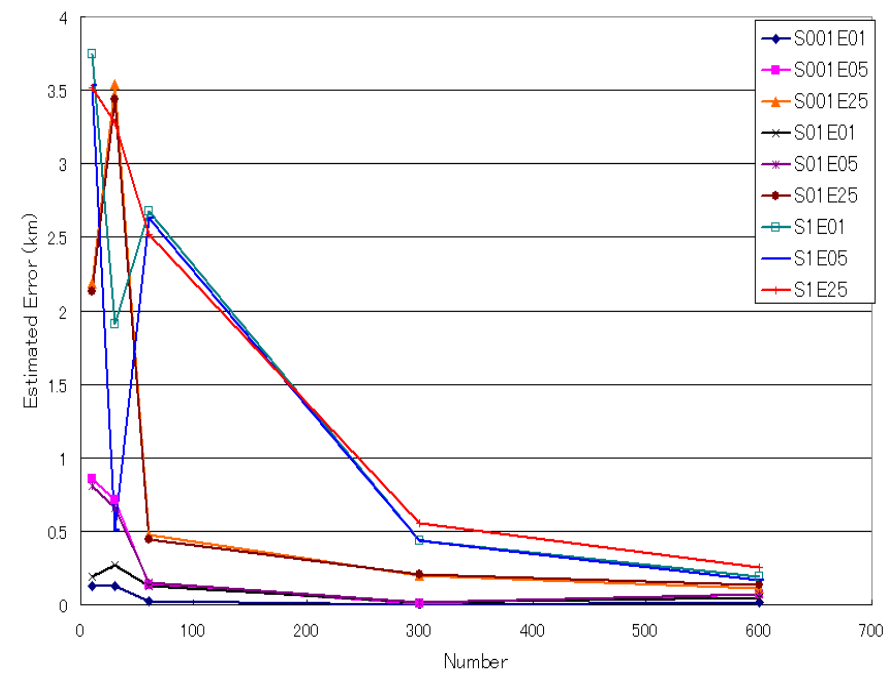

Fig. 3. Self-position estimation results (Position)

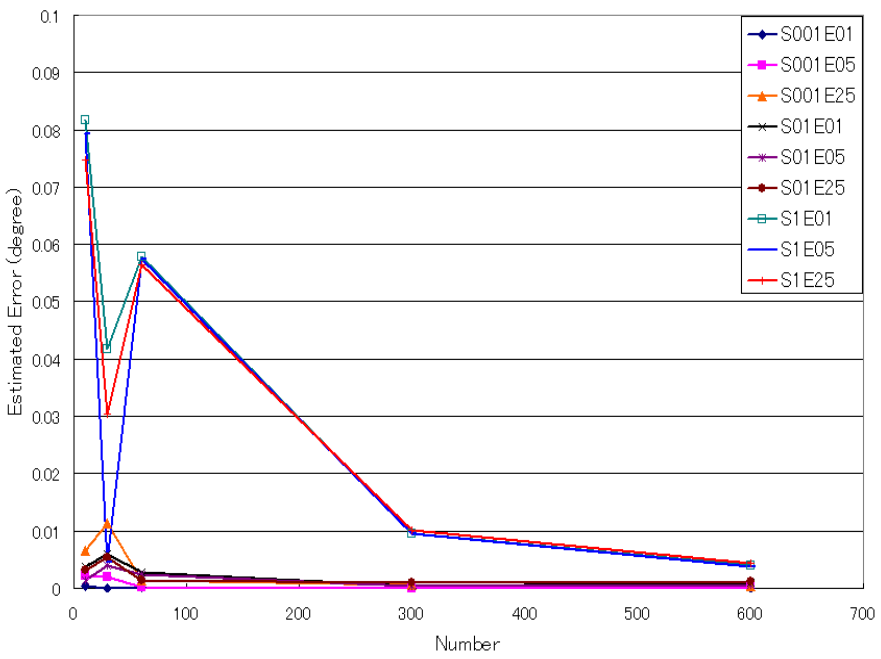

Fig. 4. Self-positin estimation results (Azimuth)

\section{Simulation Study}

The position of a rover $\left(\lambda, \phi, A_{z 0}\right)$ is estimated from the observed value $y$, which contains a measurement error by a sun sensor and an Earth sensor. The pattern of the measurement error is supposed to be normal distribution, therefore, some of errors as follows are added to the observed value. In this simulation, position is supposed to be in a central hill of the crater "Aristarchus", where a candidate in the future moon missions.

\section{The accuracy of the Sun sensor: $0.01^{\circ}, 0.1^{\circ}, 1^{\circ}(3 \sigma)$ \\ The accuracy of the Earth sensor:

$$
0.1^{\circ}, 0.5^{\circ}, 2.5^{\circ}(3 \sigma)
$$

Date: August 20, 2002.

The moon equator radius: $1738[\mathrm{~km}]$

Longitude $\lambda: 23.7 N^{\circ}$

Latitude $\phi: 47.4 W^{\circ}$

The results of the simulation are shown in Fig. 3 and Fig. 4. The position estimation is saturated when the observation of about 300 times. The estimated error is 500 meters or less. The position of less than 300 meters can be estimated with 600 times of observations. Furthermore, azimuth is estimated with accuracy of approximately $0.01^{\circ}$.

\section{ACCURATE SElF-Positioning StRATEGy}

To reduce the position error estimated by the sun and the earth observation method, it is proposed that an integrated localization method with dead reckoning. Dead reckoning is effective when a robot moves in a short distance, however, position error accumulates as a robot moves further and further. Therefore, the sensor fusion techniques are introduced to obtain the precise positioning information.

\section{A. Integration Algorithm}

At first, the position of a rover is estimated by observing the sun and the earth on the point $m$. The estimated position is defined as an expectation of the position $\bar{x}_{s}$, and existence probability around the estimated position $\bar{x}_{s}$ is supposed to be a normal distribution with dispersion $\sigma_{s}$ as:

$$
S_{k}(x) \mid x-3 \sigma_{s} \leq x \leq x+3 \sigma_{s} \quad(k=m)
$$

where subscript of $k(=1,2, \ldots m, \ldots)$ represents position number of the rover.

Next, relative position estimation from $m$ to $m+1$ is carried out by dead reckoning at all times, the estimated movement is represented as $d_{m}$. Existence probability after the movement $d_{m}$ is supposed to be a normal distribution with dispersion $\sigma_{d}$ as: 


$$
D_{k}^{j}\left(d_{k}\right) \mid d_{k}-3 \sigma_{d} \leq d_{k} \leq d_{k}+3 \sigma_{s} \quad(k=m)
$$

where subscript of $j(=1,2, \ldots)$ represents update number.

The movement from $m$ to $m+1$ is estimated by dead reckoning, however, the start point is unknown in existence range $S_{m}(x)$ of the point $m$. Assuming that the start point is in all of the range $x-3 \sigma_{s} \leq x \leq x+3 \sigma_{s}$, existence probability at position $m+1$ is shown as:

$$
D_{k}^{j}\left(x+d_{k}\right)\left(x-3 \sigma_{s} \leq x \leq x+3 \sigma_{s}\right)(k=m)
$$

Weight $W_{m}^{j}(x)$ is calculated using both $D_{k}^{j}\left(x+d_{k}\right)$ and the estimated position $S_{m+1}(x)$ on the point $m+1$ as:

$$
W_{k}^{j}(x)=P\left(D_{k}^{j}\left(x+d_{k}\right) \cap S_{k+1}(x)\right)(k=m, j=1)
$$

where $P(*)$ represents probability. Existence probability $S_{m}(x)$ of the point $m$ is updated by being multiplied the weight $W_{m}^{j}(x)$ as:

$$
L_{k}^{j}(x)=C\left(W_{k}^{j}(x) \times S_{k}(x)\right)(k=m)
$$

where $C$ is a coefficient for regularization. After that, weight is defined as:

$$
\begin{array}{r}
W_{k}^{j}(x)=P\left(D_{k}^{j}\left(x+d_{k}\right) \cap L_{k+1}^{j}(x)\right) \\
\quad(k=m-1, m-2, \ldots, j=2,3, \ldots)
\end{array}
$$

According the above equations, the positions estimated previously are updated backward, so we call the fusion method "backward propagation". The image of the proposed algorithm is shown in Fig. 5.

The backward propagation can only improve the precision of estimation of past position, however, the proposed algorithm can be applicable for the present position to change some subscripts:

$$
\begin{aligned}
& W_{k+1}^{j}(x)=P\left(D_{k}^{j}\left(x+d_{k}\right) \cap S_{k}(x)\right)(k=m, j=1) \\
& L_{k+1}^{j}(x)=C\left(W_{k+1}^{j}(x) \times S_{k+1}(x)\right)(k=m) \\
& W_{k+1}^{j}(x)=P\left(D_{k}^{j}\left(x+d_{k}\right) \cap L_{k}^{j}(x)\right)(k=m, j=2,3, \ldots)
\end{aligned}
$$

We call this operation "forward propagation", because it updates the estimation one after another with past data.

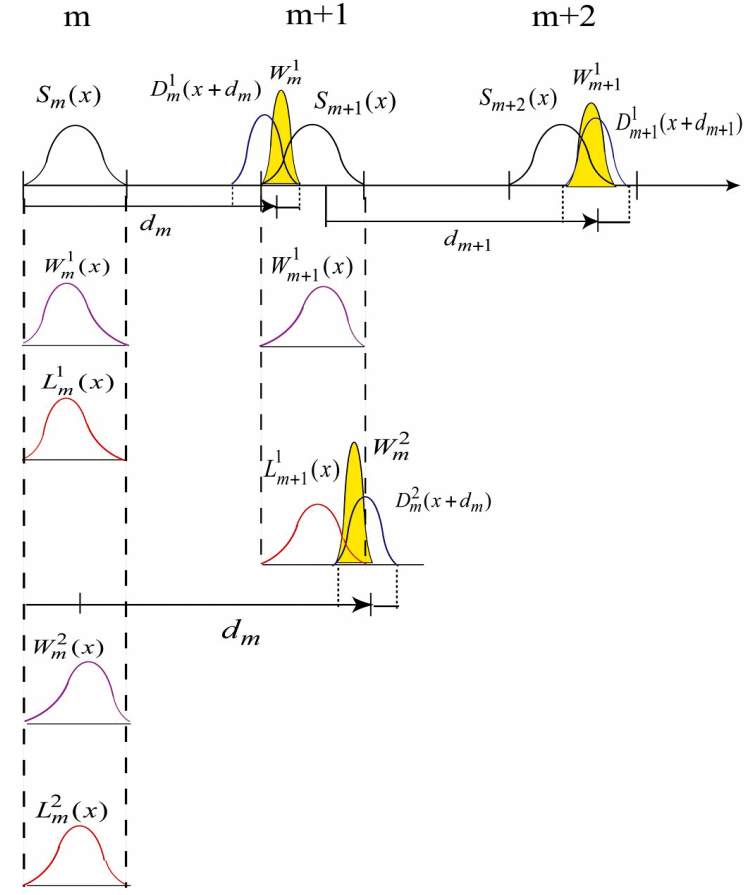

Fig. 5. Fusion image (Backward propagation)

\section{B. Simulation Study 1 (investigation of statistical validity)}

To investigate the effectiveness of LAR, a numerical simulation is performed. Parameters in this simulation are set as follows.

True position interval;

$300,600,900,1200,1500,1800[\mathrm{~m}]$

The accuracy of the sun $\&$ the earth observation;

$150[m](3 \sigma)$

The accuracy of dead reckoning;

$10 \%$ of movement distance

$$
30,60,90,120,150,180[\mathrm{~m}](3 \sigma)
$$

The normal distribution errors were added to the observation values.

Monte Carlo simulation (300 times) was performed in order to confirm the statistical validity of an estimated value. The backward propagation result is shown in Fig. 6, the forward propagation result is shown in Fig. 7. Then, the simulation results of integration updates in case of 300 meters interval are shown in Fig 8.

Fig. 6 and Fig. 7 show that the error of estimated value is saturated within five times of updates. Then, LAR can estimate the positions twice as precise as only the sun and earth observation. Fig. 8 shows that an older measurement value has more number of times of updating with back propagation, and a newer measurement value has more number of times of updating with forward propagation. There are shown that estimation accuracy is improved as backward propagation and forward propagation are carried out. 


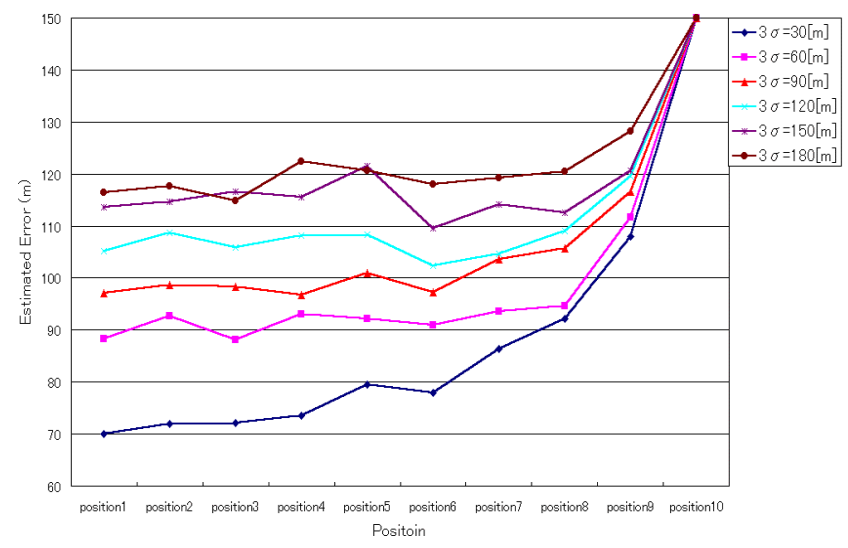

Fig. 6. Monte Carlo simulation results (Backward propagation)

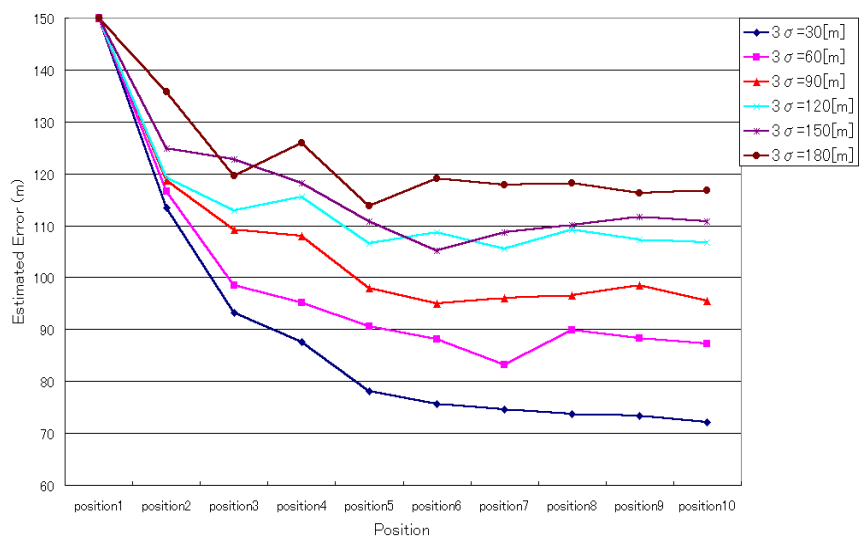

Fig. 7. Monte Carlo simulation results (Forward propagation)
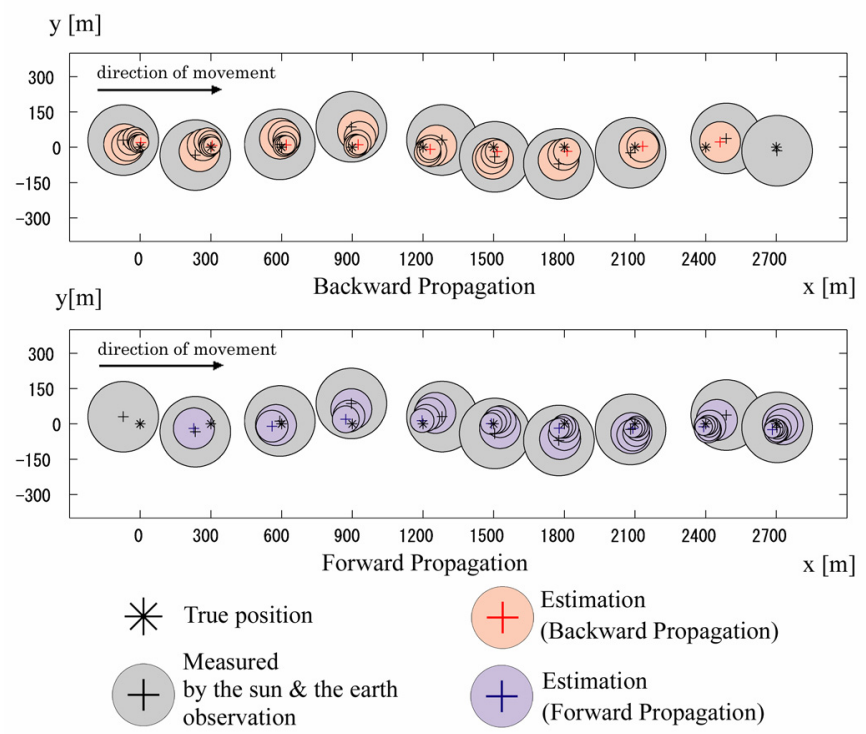

Fig. 8. Simulation results of integration updates

\section{Simulation Study 2 ( comparison with EKF)}

LAR can improve an accuracy of position estimation by integrating two or more sensor information. It is similar to EKF's approach. EKF is a well-known sensor fusion technique, and its validity has been shown in robotistic field [9]. In this section, in order to compare LAR with EKF, a simulation which is supposed on long-distance moving on the moon was carried out.

\section{Assumptions of simulation}

Since supposed mission term on the moon is limited for two weeks because of length of daytime of the moon, and a rover on the moon has to run for a few kilometers, following assumptions are considered.

(1) The rover runs for hundreds of hours stably. The running speed of the rover should be extremely slow (approx. $0.1[\mathrm{~m} / \mathrm{s}]$ ) because of its energy consumption, the rover is supposed to keep running for 300 hours in one time.

(2) The rover cannot measure its absolute position frequently. In order to measure its absolute position accurately by the sun and earth observation, the rover must stop while the measurement is carried out. Therefore, the condition that the rover acquires an absolute position is set as one time for every 3 hours.

(3) Velocity of the rover is estimated by wheel encoders, and relative azimuth is measured by rate gyros. The rover updates its estimated position by dead reckoning every $0.1[\mathrm{~s}]$.

In the following simulation, the normal distribution errors of the observed positions are supposed as follows.

$$
\begin{aligned}
& \text { The sun } \& \text { the earth sensor; } \\
& 3 \sigma=150[\mathrm{~m}] \\
& \text { Wheel-encoder; } \\
& 3 \sigma=3.0 \times 10^{-2}[\mathrm{~m} / \mathrm{s}] \\
& \text { Rate gyros; } \\
& 3 \sigma=1.75 \times 10^{-2}[\mathrm{rad}]
\end{aligned}
$$

\section{LAR vs. EKF}

When the position estimation is carried out by LAR, number of updates (back and forward propagation) should be determined. As the results of the simulation in previous section, five times of updates should be enough. In the following simulation, forward propagation is used.

In EKF, following models are used.

$$
\begin{aligned}
& \left\{\begin{array}{l}
x_{t+1}=x_{t}+\left(v_{t} \cos \theta_{t}+\rho_{t} \cos \theta_{t}-\phi_{t} v_{t} \sin \theta_{t}\right) \Delta \\
y_{t+1}=y_{t}+\left(v_{t} \sin \theta_{t}+\rho_{t} \sin \theta_{t}+\phi_{t} v_{t} \cos \theta_{t}\right) \Delta
\end{array}\right. \\
& \left\{\begin{array}{l}
\xi_{c(i)}=x_{c(i)}+\delta_{c(i)} \\
\eta_{c(i)}=y_{c(i)}+\varepsilon_{c(i)}
\end{array}\right.
\end{aligned}
$$




\author{
$v_{t}:$ Velocity of rover \\ $\rho_{t}:$ Measurement error of velocity \\ $\theta_{t}:$ Azimuth of rover \\ $\phi_{t}:$ Measurement error of azimuth \\ $\Delta:$ Sampling interval \\ $\left(\xi_{t}, \eta_{t}\right)$ : Absolute position from the Sun \& Earth observation \\ $\left(\delta_{t}, \varepsilon_{t}\right):$ Measurement error of absolute position \\ $c_{(i)}:$ Time to get absolute position
}

To simplify the simulation, we suppose the rover goes straight at the rate of $0.1[\mathrm{~m} / \mathrm{s}]$ for 300 hours.

The results of position estimation of both methods are shown in Fig. 9. RMS errors of the estimated positions of both methods are shown in Fig. 10. In the results of EKF, more than 80 times of measurements of absolute positions were required on the condition of the simulation. 80 times of measurements correspond to $80,000[\mathrm{~m}]$ of traverse. Therefore, in order to saturate the estimation by EKF, at least 80,000 [m] of traverse is required. In this application, since relative and absolute positions are integrated, the relative positions have to be measured. In other words, if the rover stopped, the integration could not be carried out - the rover must move during the time for convergence.

Since the time of the lunar exploration is limited, the rover cannot use the time for converging the position estimation. As shown in section III, the algorithm of LAR doesn't need time for convergence. Therefore, LAR is incomparably better than EKF in this application. It should be noted that if the time to explore were unlimited, EKF could estimate the positions more precise than LAR.

RMS error of LAR is saturated within five times of updates, and after saturation, the estimated value is stable. The standard deviations of estimates by LAR and EKF were 38.83 [m] and $87.75[\mathrm{~m}]$ respectively when the position estimation was saturated. As the result of the simulation, LAR can converge RMS aprox.15 times faster than EKF, and can estimate absolute positions two times as precise as those of EKF on the moon.

\section{CONCLUSIONS}

This paper presented a method to estimate absolute position of a lunar rover by using the sun sensor and the earth sensor. This paper also proposed an accurate localization scheme named "Localization in combination with Absolute and Relative position (LAR)", which is to integrate the sun and the earth observation and the dead reckoning. The effectiveness of LAR was confirmed by some numerical simulations. Moreover, if certain absolute position sensor, e.g. GPS, was substituted for the sun and the earth sensor to estimate absolute position, we could use the method for mobile robots on the earth.

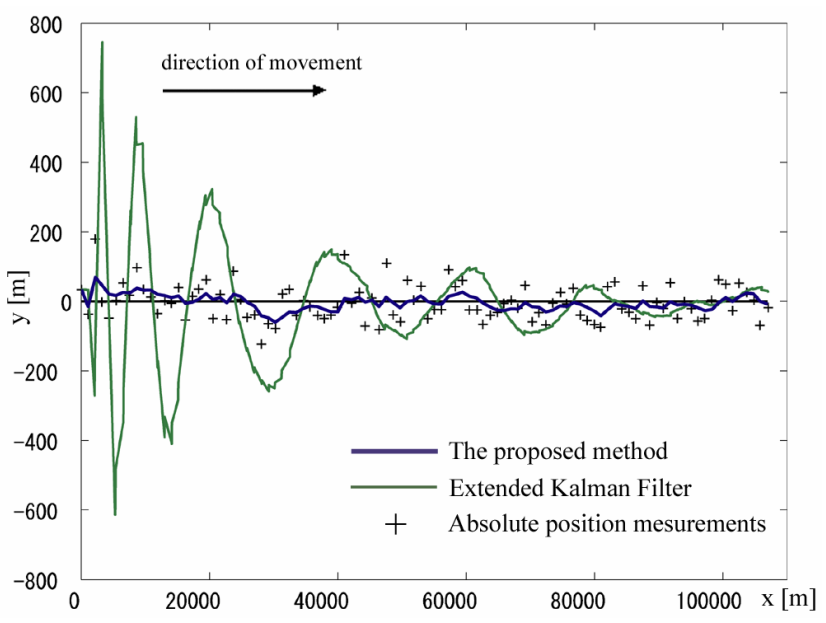

Fig. 9. Estimated trajectory of the rover by each method

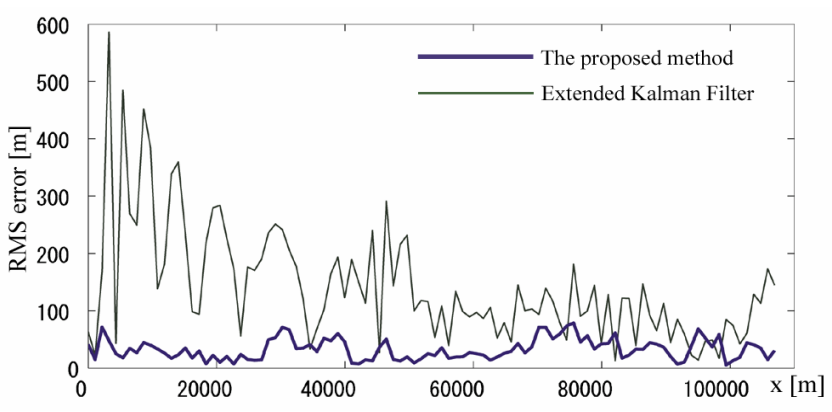

Fig. 10. RMS errors of each estimation

\section{REFERENCES}

[1] Y.Kuroda, K.Kondo, T.Miyata, M.Makino, "The Micro5 Suspension System for Small Long range Planetary Rover", Proc. of ISAS 8th Workshop on Astrodynamics and Flight Mechanics, pp.287-292, 1998.

[2] J.E.Potter and W.E.Vander Velde: "Optimum mixing of gyroscope and star tracker data", J. of Spacecraft and Rockets5, pp.536-540, May 1968.

[3] Borenstein, L.Feng: "Gyrodometry: A new method for combining data from gyros and odometry in mobile roots", Proc. of IEEE Int'l Conf. on Robotics and Automation, pp.569-574, 1996.

[4] J.C.Alexander and J.H.Maddocks, "On the Kinematics of Wheeled Mobile Robots", J. of Robotics Research, 1990.

[5] Tonouchi, Tsubouchi, Arimoto, "A Position Estimation which Takes Account of a Closed Space Model for a Mobile Robot - A Bayesian Fusion Method Using Internal Sensory Data and Knowledge about Work Space-lk", J. of the Robotics Society of Japan Vol.12 No.5, pp.695-699, 1994.

[6] I.Nakatani, T.Kubota, T.Yoshimitsu: "Position Estimation for Planetary Rover by Observation of the Sun", Proc. of the 37th Space Sciences and Technology Conference, pp.369-370, 1993.

[7] A.Tsuchiya, T.Kubota, Y.Kuroda, T.Yoshimitsu: "A Method to Estimate Position and Azimuth for Lunar Rover", Proc. of 2000 JSME Conference on Robotics and Mechatoronics, 2P1-09-008, 2000.

[8] A.Tsuchiya, T.Kubota, Y.Kuroda, T.Yoshimutsu: "A Method to Estimate Absolute Position and Azimuth of Lunar Rover by using Sun Sensor and Earth Sensor", Proc. of ISAS 11th Workshop on Astrodynamics and Flight Mechanics, pp.240-245, 2001.

[9] Stergios.I.Roumeliotis, George A.Bekey: "An Extended Kalman Filter for frequent local and infrequent global sensor data fusion", Proc. of SPIE, Vol.3209, 1997. 